\title{
Prosody Learning Mechanism for Speech Synthesis System Without Text Length Limit
}

\author{
Zhen Zeng, Jianzong Wang*, Ning Cheng, Jing Xiao \\ Ping An Technology (Shenzhen) Co., Ltd. \\ \{zengzhen830, wangjianzong347, chengning211, xiaojing661\}@pingan.com.cn
}

\begin{abstract}
Recent neural speech synthesis systems have gradually focused on the control of prosody to improve the quality of synthesized speech, but they rarely consider the variability of prosody and the correlation between prosody and semantics together. In this paper, a prosody learning mechanism is proposed to model the prosody of speech based on TTS system, where the prosody information of speech is extracted from the melspectrum by a prosody learner and combined with the phoneme sequence to reconstruct the mel-spectrum. Meanwhile, the sematic features of text from the pre-trained language model is introduced to improve the prosody prediction results. In addition, a novel self-attention structure, named as local attention, is proposed to lift this restriction of input text length, where the relative position information of the sequence is modeled by the relative position matrices so that the position encodings is no longer needed. Experiments on English and Mandarin show that speech with more satisfactory prosody has obtained in our model. Especially in Mandarin synthesis, our proposed model outperforms baseline model with a MOS gap of 0.08 , and the overall naturalness of the synthesized speech has been significantly improved.
\end{abstract}

Index Terms: speech synthesis, text-to-speech, self-attention, prosody modeling

\section{Introduction}

Speech synthesis, also known as text-to-speech (TTS), has attracted a lot of attention and obtained satisfactory results in recent years due to the advances in deep learning. Several TTS systems based on deep networks were proposed, such as Char2Wav [1], Tacotron2 [2], DeepVoice3 [3], Transformer TTS [4], FastSpeech [5] and ParaNet [6]. These systems usually first predict the acoustic feature sequence from the input text sequence, and then generate waveform from the acoustic feature sequence using vocoder such as Griffin-Lim [7], WaveNet [8], WaveRNN [9], WaveGlow [10] and GAN-TTS [11].

Although current speech synthesis systems have obtained high-quality speech, it is difficult for them to achieve satisfactory results in long text speech synthesis scenarios. In the sequence-to-sequence TTS model, since the monotonicity and locality properties of TTS alignment are not fully utilized, the alignment procedure lacks robustness in inference, which leads to skipping or repeating words, incomplete synthesis, or an inability to synthesize long utterances [12]. To address the issue, many monotonic attention mechanisms are presented [13, 14], where only the alignment paths satisfying the monotonic condition are taken into consideration at each decoder timestep. In [12. 15], the location-based GMM attention introduced in [16] is also studied in TTS systems to generalize to long utterances.

\footnotetext{
*Corresponding author: Jianzong Wang, jzwang@188.com
}

Especially, AlignTTS [17] proposes an alignment loss to model the alignment between text and mel-spectrum, and uses a length regulator to adjust the alignment, which solves the instability problem of the alignment and is very efficient. However, since the self-attention of Transformer [18] is used to model the dependencies of input sequence elements in AlignTTS, the positional encodings are required to introduce the positional information, which limits the maximum length of input text. In this paper, a novel self-attention mechanism is proposed to remove the need for the positional encodings and lift the restriction of input text length.

On the other hand, the prosody of speech directly affects the overall listening perception of the voice, especially for long utterances. In order to improve the naturalness of synthetic speech, it is necessary for TTS systems to model prosody information. In [19], a prosody embedding is introduced for emotional and expressive speech synthesis, which enables finegrained control of the speaking style. In [20], an interpretable latent variable model for prosody based on Tacotron 2 is presented to model phoneme-level and word-level prosody information of speech. [21] proposes a quantized latent feature for the prosody of speech, and trains an autoregressive prior model to generate natural samples without a reference speech. These prosody control methods enable us to learn the prosody from speech and fine-grained the synthesized speech, but they still cannot effectively predict the correct prosody according to the input text. One reason is that the prosody information of speech generally depends on the meaning of text, while only the phoneme information of text is used as the input in current mainstream TTS systems, which limits the capabilities of modeling the prosody of speech. In [22, 23, 24], the textual knowledge from BERT [25] is introduced into TTS systems to improve the prosody of speech, but they ignore the variability of prosody. For example, the same text may produce speech with different prosody due to pronunciation uncertainty.

In this works, we propose a novel self-attention mechanism, named as local attention, to model the timing dependencies, which abandons positional encoding and uses a relative position matrix to model the influence of the positional relationship of input sequence. At the same time, we introduce the prosody learning mechanism for feed-forward TTS systems, where a prosody embedding for each phoneme is learned from the mel-spectrum in training. In addition, a prosody predictor is designed to predict the prosody embedding according to text and phoneme, where a pre-trained language model is applied to introduce the meaning of text. And the main contributions of our works as follows:

- The local attention is proposed to model sequence dependency so that the speech synthesis system is no longer restricted by the length of the input text sequence;

- The prosody learning mechanism is designed for feed- 
forward TTS system to capture the prosody information from the mel-spectrum. Thanks to the separation of the prosody information, more accurate mel-spectrum prediction model is obtained;

- In order to improve the prosody prediction results, the pre-trained language model is applied in TTS system to introduce the sematic features of text.

\section{Our Methods}

In this section, we propose the local attention that can capture the sequence dependency without the positional encoding, and the prosody learning mechanism that can separate the prosody of speech from the pronunciation of phoneme. And based on the architecture of AlignTTS, a feed-forward speech synthesis system with prosody learning ability is designed, which has not limit on the length of input text.

\subsection{Local Attention Block}

\subsubsection{Self-Attention of Transformer}

Recent years, the self-attention mechanism is proposed to capture the context-sensitive features in Transformer [18], which is widely used in various nature language process task 25 26, 27, 28]. Due to the similarity of machine translation and speech synthesis, the structure of Transformer is also applied in TTS systems, such as Transformer TTS [4], FastSpeech [5] and AlignTTS [17]. Although the self-attention structure has been proven to achieve satisfactory performance in speech synthesis, it is not perfect for TTS system. In detail, the self-attention is designed to model the dependence of any two elements in the input sequence, which does not make good use of the law of the influence of the input sequence position distance relationship on pronunciation. On the other hand, the positional encodings are used to inject the positional information of input sequence, but it also limits the maximum length of the input sequence.

\subsubsection{Local Attention}

In our works, we design a novel self-attention network, named as local attention, which is more suitable for TTS systems. Let $\boldsymbol{h}=\left\{h_{1}, h_{2}, \ldots, h_{n}\right\}$ denote the input sequence, where each element in sequence is vector. The attention function is described as mapping a query and a set of key-value pairs to an output. Following the Transformer, the query sequence, the key sequence and the value sequence are calculated by

$$
\boldsymbol{q}, \boldsymbol{k}, \boldsymbol{v}=\boldsymbol{h} \mathbf{W}^{q}, \boldsymbol{h} \mathbf{W}^{k}, \boldsymbol{h} \mathbf{W}^{v}
$$

Different from Transformer using the dot products of $\boldsymbol{q}$ and $\boldsymbol{k}$ to compute the attention weights, the local attention introduces a relative position matrix to model the positional relationship between elements in $\boldsymbol{q}$ and $\boldsymbol{k}$. The attention weights before softmax function can be calculated by

$$
A_{i, j}=\left\{\begin{array}{cc}
\left(q_{i}\right)^{\top} \mathbf{W}_{(i-j)}^{\mathrm{loc}} k_{j} & |i-j| \leq T \\
-i n f & |i-j|>T
\end{array}\right.
$$

where $\mathbf{W}^{\text {loc }}=\left\{\mathbf{W}_{-T}^{\text {loc }}, \ldots, \mathbf{W}_{0}^{\text {loc }}, \ldots, \mathbf{W}_{T}^{\text {loc }}\right\}$ denotes the relative position matrices, and $T$ denotes the maximum relative position distance considered by the attention calculation. And the local attention can be written as

$$
\text { Local-Attention }(\boldsymbol{h})=\operatorname{Masked-Softmax}\left(\frac{\mathbf{A}}{\sqrt{d_{k}}}\right) \boldsymbol{v}
$$

where $\mathbf{A}=\left\{A_{i, j}\right\}_{n \times n}$, and $\sqrt{d_{k}}$ is the scaling factor.

\subsubsection{Network Structure}

Similar to Transformer, the multi-head mechanism is also adopted in the local attention. In addition, the local attention block is designed to facilitate multi-layer stacking, which is composed of a multi-head local attention and a 2-layer 1D convolution network. The residual connection [29], layer normalization [30] and dropout [31] are also used, same as Transformer. Note that, in the local attention, the relative positional information can be modeled by the relative position matrices $\mathbf{W}^{\mathrm{loc}}$, thus the positional encodings can be removed. In our opinion, compared with the positional encodings, the relative position matrices make the network more capable of modeling the location information.

\subsection{Prosody Modeling}

\subsubsection{Prosody Learning}

The prosody of speech affects the listening sensation of the entire speech, especially for long text speech. To obtain synthetic speech that approximates a real person, prosody information must be modeled when synthesizing speech of long text. Meanwhile, using different prosodies to speak, the same text will obtain different speech, thus it is necessary for advanced TTS systems to consider the effect of prosody on pronunciation.

In our works, we propose the prosody learning mechanism to model the prosody of speech in training. As shown in Figure 1(a) a prosody learner is designed to calculate the prosody embedding according to the mel-spectrum, which is added into phoneme embedding to affect the training of TTS systems. In detail, we first calculate the alignment between the phoneme sequence and the mel-spectrum sequence using the alignment loss introduced in AlignTTS [17]. The mel-spectrum sequence is passed through multi-layer 1D convolutional network, where residual connection and relu activation function are used, and then it is pooled according to the alignment between phoneme and mel-spectrum to output a prosody representation sequence. The prosody representation sequence is low-dimensional (for example, 3), of which length is equal to the length of the phoneme sequence. In addition, a prosody mapping network, just a linear layer, is designed to generate prosody embedding from the prosody representation sequence. The prosody embedding is added into the phoneme embedding in the mel-spectrum predictor and the duration predictor.

\subsubsection{Prosody Prediction}

In our daily speech, the prosody of each phoneme is often more corresponding to the meaning of the word. Therefore, in order to predict more satisfactory prosody, we introduce the pre-trained language model to design a prosody predictor. As shown in Figure 1(b) the prosody predictor is composed of the phoneme embedding layer, the pre-trained language model, and multiple local attention blocks and linear layers. The word embeddings from the pre-trained language model is first upsampled to align with the phoneme sequence. The phoneme embedding is passed through 3-layer 1D convolution networks, and the is added to the upsampled word embedding. Multiple stacked local attention blocks are used to model the dependencies between sequence elements. And the last linear layer outputs the distribution of the prosody representation. Considering the variability of prosody, the mix density network [32] is used to model the distribution of the prosody representation. In detail, the last linear layer outputs the mean, variance and weight of multiple mixed Gaussian distributions for each element of the prosody 


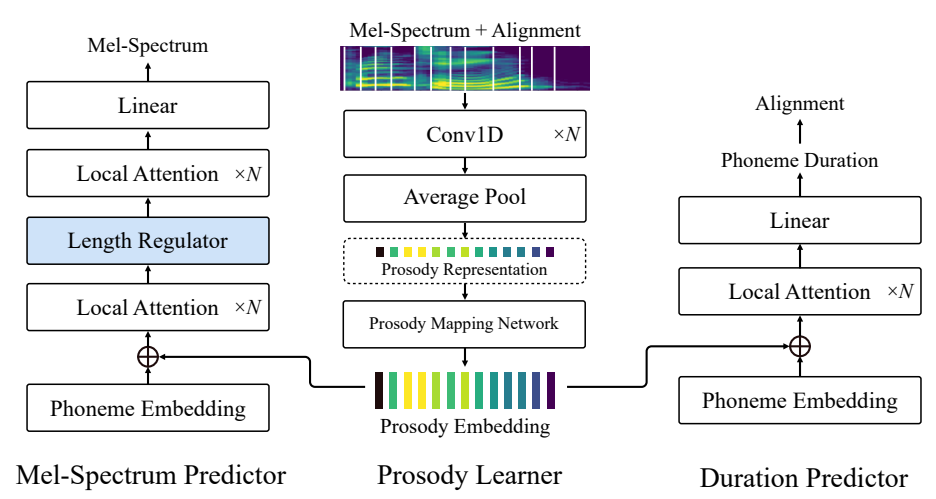

(a) Model Training with Prosody Learning Mechanism

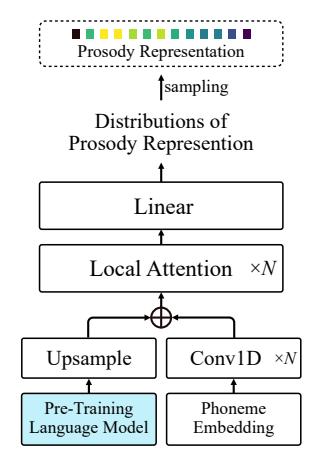

Prosody Predictor

(b) Prosody Predictor

Figure 1: (a) Model training with prosody learning mechanism, where a prosody learner is designed to model the prosody information from mel-spectrum of speech in training dataset. (b) Prosody predictor, where a pre-training language model is introduced to improve the prosody prediction results.

representation sequence.

In order to train the prosody predictor, we need first finish the training of the prosody learner, and use it to generate the prosody representation sequence of speech in training dataset. The generated prosody representation sequence is used as the target to guide the training of the prosody predictor.

\subsection{Text-to-Speech System}

\subsubsection{Architecture}

Using the local attention and the prosody modeling, we design a feed-forward TTS system based on AlignTTS. As shown in Figure 11, the proposed system consists of a mel-spectrum predictor for transforming the phoneme sequence into the melspectrum, a duration predictor for predicting the duration of each phoneme, a prosody learner for modeling the prosody of speech, and a prosody predictor for predicting the prosody in inference. The mel-spectrum predictor and the duration predictor are modification version of AlignTTS, where the FFT block of AlignTTS is replaced by the local attention block and the positional encoding is removed. The length regulator is the same as that in AlignTTS, which requires correct alignment between phonemes and mel-spectrum in training and the phoneme durations predicted by the duration predictor in inference.

\subsubsection{Training}

In training, we first model and extract the alignment between phonemes and mel-spectrum according to [17]. The melspectrum predictor, the duration predictor and the prosody learner are trained together, where the extracted alignment is used to guide the average pooling in prosody learner and converted into the duration sequence as the target of the duration predictor. After finishing their training, the prosody representation sequence of each speech in training dataset is calculated by the trained prosody learner, which is used as the target to guide the training of the prosody predictor.

\subsubsection{Inference}

In inference, according to the text and its phonemes, the prosody predictor first predicts the distributions of the prosody representation. The prosody representation sequence sampled from the predicted distributions is passed through the prosody mapping network to generate the prosody embedding that is added into the phoneme embedding to predict the duration sequence in the duration predictor. Based on the prosody embedding and the duration sequence, the mel-spectrum predictor generates the mel-spectrum. Finally, A neural vocoder, such as WaveGlow [10], is used to generate the waveform according to the predicted mel-spectrum.

\section{Experiments}

\subsection{Dataset}

We test the performance of our model in synthesizing English and Mandarin. The LJSpeech dataset [33] is chosen as the English speech dataset, which consists of 13100 short audio clips of a single speaker and corresponding transcriptions. The Mandarin speech dataset uses the Chinese Standard Mandarin Speech Copus from Databaker [34], which is composed of 10000 speech of a female and transcriptions. These datasets are randomly divided into 2 sets: $98 \%$ of samples for training and the rest for testing. The sample rate of audio is set to $22050 \mathrm{~Hz}$. The mel-spectrums are computed through a shorttime Fourier transform with Hann windowing, where 1024 for FFT size, 1024 for window size and 256 for hop size. The STFT magnitude is transformed to the mel scale using 80 channel mel filter bank spanning $60 \mathrm{~Hz}$ to $7.6 \mathrm{kHz}$.

For English synthesis, the characters of text are directly used as the input of model, and for Mandarin synthesis, the pinyin sequence of text is used as input. In addition, in order to verify the prosody modeling capabilities, all marks of rhythm boundaries are replaced by spaces in our experiences.

\subsection{Model Configuration}

In our experiments, the number of the local attention block is set to 6 in the alignment learner. The mel-spectrum predictor contains 6 local attention blocks on both the phoneme side and the mel-spectrum side, and the duration predictor includes 3 local attention blocks. The channel of these network is all set to 768 . In all local attention blocks, the number of attention head is set to 2 , the kernel size of $1 \mathrm{D}$ convolution is set to 3 , and the maximum relative position distance of the relative position matrices is set to 10 . The number of the 1D convolution layers is 4 in the prosody learner, and the number of the local attention blocks is 
Table 1: The comparison of MOS among different TTS systems.

\begin{tabular}{llc}
\hline Language & Method & MOS \\
\hline \multirow{4}{*}{ English } & Ground Truth & $4.56 \pm 0.05$ \\
& Tacotron 2 & $3.96 \pm 0.13$ \\
& FastSpeech & $3.88 \pm 0.11$ \\
& AlignTTS & $4.05 \pm 0.12$ \\
& Ours & $\mathbf{4 . 0 5} \pm \mathbf{0 . 0 6}$ \\
\hline \multirow{4}{*}{ Mandarin } & Ground Truth & $4.68 \pm 0.05$ \\
& Tacotron 2 & $3.94 \pm 0.10$ \\
& FastSpeech & $3.81 \pm 0.08$ \\
& AlignTTS & $3.95 \pm 0.11$ \\
& Ours & $\mathbf{4 . 0 3} \pm \mathbf{0 . 0 9}$ \\
\hline
\end{tabular}

also 4 in the prosody predictor. In addition, AlBert [35] is chosen as the pre-trained language model, and we directly use the official open-source trained model in our experiments.

Our TTS system is trained on 2 NVIDIA V100 GPUs with batch size of 16 samples on each GPU. The Adam optimizer [36] with $\beta_{1}=0.9, \beta_{2}=0.98, \varepsilon=10^{-9}$ is used in our case. In addition, we adopt the same learning rate schedule described in [18] in whole training.

\subsection{Results}

In order to evaluate the performance of our model, we compare the quality of speech synthesized by Tacotron 2 [2], FastSpeech [5], AlignTTS [17] and our model. In details, the text transcription in test datasets is used as the input of these model to predict the mel-spectrum, and then the WaveGlow vocoder is used to generate the waveform from the predicted mel-spectrum. The audios synthesized by different models are rated together with the ground truth audio (GT) by 50 testers. And then the mean opinion score (MOS) is calculated as the evaluation indicator.

The evaluation results are shown in Table 1 , where the synthetic speech of English and Mandarin are rated separately. It can be seen that our model gets the same performance as AlignTTS, and outperforms Tacotron 2 by 0.09 in synthesizing English. In Mandarin experiments, our model obtains significantly improved performance than AlignTTS. According to our analysis, the synthetic speech from our model has more satisfactory prosody, which makes the overall listening experiences of speech greatly improved, especially for Mandarin.

\subsection{Ablations}

\subsubsection{Pre-trained Language Model}

In our model, the pre-trained language model is introduced to predict the prosody information. In order to verify the effectiveness, we conduct a comparative experiment on whether the prosody predictor needs information from the pre-trained language model. A comparative prosody predictor without the pre-trained language model is trained and predict the prosody presentation for synthesizing speech. These speech predicted by different prosody predictor are rated together, and the results are shown in Table 2. We can see that the introduction of the pre-trained language model can significantly improve the quality of synthetic speech, especially in prosody. Meanwhile, even without the pre-trained language model in prosody predictor, our model can still obtain better performance than AlignTTS, which verifies that the separation of prosody information is also
Table 2: The effect of the pre-trained language model on Mandarin synthesis.

\begin{tabular}{lc}
\hline Method & MOS \\
\hline AlignTTS & $3.95 \pm 0.11$ \\
Without Pre-trained Language Model & $3.97 \pm 0.11$ \\
With Pre-trained Language Model & $4.03 \pm 0.09$ \\
\hline
\end{tabular}

Table 3: The effect of the dimension of the prosody representation on Mandarin synthesis.

\begin{tabular}{lc}
\hline Dimension & MOS \\
\hline 1 & $3.98 \pm 0.12$ \\
3 & $4.03 \pm 0.09$ \\
6 & $4.02 \pm 0.05$ \\
10 & $3.99 \pm 0.13$ \\
\hline
\end{tabular}

beneficial to the mel-spectrum modeling.

\subsubsection{Dimension of Prosody Representation}

In order to model the prosody information, we introduce the prosody representation that is learned from speech in training process and is predicted by the prosody predictor in inference process. The prosody representation is a compressed representation sequence of speech, which can be used to reconstruct speech when providing the phoneme sequence. Therefore, the dimension of the prosody representation is a significant hyperparameter, since too high dimension will introduce too much information from the speech, which affects the modeling of the pronunciation of each phoneme in the mel-spectrum predictor. We experimentally analyze the influence of this dimension on the performance of TTS system. The synthetic speech from different TTS systems with different dimensions of the prosody representation are rated by our tester together. The results are shown in Table 3 It can be seen that appropriate dimension can make the model get the best performance, and too high dimension reduces model performance.

\section{Conclusion}

Based on the local attention, a feed-forward text-to-speech system without the limitation of input text length is designed. Meanwhile, the prosody learning mechanism is proposed to model the prosody of speech, where the prosody information is learned from speech by a prosody learner in training process. In order to predict more satisfactory prosody in inference, the pre-trained language model is used to introduce the semantic feature. Experiments on English synthesis and Mandarin synthesis show that a significant improvement in the prosody of speech has been obtained in our proposed TTS systems.

\section{Acknowledgements}

This paper is supported by National Key Research and Development Program of China under grant No. 2018YFB1003500, No. 2018YFB0204400 and No. 2017YFB1401202. Corresponding author is Jianzong Wang from Ping An Technology (Shenzhen) Co., Ltd. 


\section{References}

[1] J. Sotelo, S. Mehri, K. Kumar, J. F. Santos, K. Kastner, A. Courville, and Y. Bengio, "Char2wav: End-to-end speech synthesis," in ICLR Workshop, 2017.

[2] J. Shen, R. Pang, R. J. Weiss, M. Schuster, N. Jaitly, Z. Yang, Z. Chen, Y. Zhang, Y. Wang, R. Skerrv-Ryan et al., "Natural tts synthesis by conditioning wavenet on mel spectrogram predictions," in International Conference on Acoustics, Speech and Signal Processing (ICASSP), 2018.

[3] W. Ping, K. Peng, A. Gibiansky, S. O. Arik, A. Kannan, S. Narang, J. Raiman, and J. Miller, "Deep voice 3: 2000-speaker neural text-to-speech," in International Conference on Learning Representations (ICLR), 2018.

[4] N. Li, S. Liu, Y. Liu, S. Zhao, M. Liu, and M. Zhou, "Neural speech synthesis with transformer network," in AAAI Conference on Artificial Intelligence (AAAI), 2019.

[5] Y. Ren, Y. Ruan, X. Tan, T. Qin, S. Zhao, Z. Zhao, and T.-Y Liu, "Fastspeech: Fast, robust and controllable text to speech," in Advances in neural information processing systems (NIPS), 2019

[6] K. Peng, W. Ping, Z. Song, and K. Zhao, "Parallel neural text-tospeech," arXiv preprint arXiv:1905.08459, 2019.

[7] D. Griffin and J. Lim, "Signal estimation from modified shorttime fourier transform," IEEE Transactions on Acoustics, Speech, and Signal Processing, vol. 32, no. 2, pp. 236-243, 1984.

[8] A. van den Oord, S. Dieleman, H. Zen, K. Simonyan, O. Vinyals, A. Graves, N. Kalchbrenner, A. Senior, and K. Kavukcuoglu, "Wavenet: A generative model for raw audio," in 9th ISCA Speech Synthesis Workshop, 2016.

[9] N. Kalchbrenner, E. Elsen, K. Simonyan, S. Noury, N. Casagrande, E. Lockhart, F. Stimberg, A. van den Oord S. Dieleman, and K. Kavukcuoglu, "Efficient neural audio synthesis," in International Conference on Machine Learning (ICML), 2018.

[10] R. Prenger, R. Valle, and B. Catanzaro, "Waveglow: A flow-based generative network for speech synthesis," in International Conference on Acoustics, Speech and Signal Processing (ICASSP), 2019.

[11] M. Bikowski, J. Donahue, S. Dieleman, A. Clark, E. Elsen, N. Casagrande, L. C. Cobo, and K. Simonyan, "High fidelity speech synthesis with adversarial networks," in International Conference on Learning Representations (ICLR), 2020.

[12] E. Battenberg, R. Skerry-Ryan, S. Mariooryad, D. Stanton, D. Kao, M. Shannon, and T. Bagby, "Location-relative attention mechanisms for robust long-form speech synthesis," in International Conference on Acoustics, Speech and Signal Processing (ICASSP), 2020.

[13] J.-X. Zhang, Z.-H. Ling, and L.-R. Dai, "Forward attention in sequence-to-sequence acoustic modeling for speech synthesis," in International Conference on Acoustics, Speech and Signal Processing (ICASSP), 2018.

[14] M. He, Y. Deng, and L. He, "Robust sequence-to-sequence acoustic modeling with stepwise monotonic attention for neural tts," in Annual Conference of the International Speech Communication Association (Interspeech), 2019.

[15] K. Kastner, J. F. Santos, Y. Bengio, and A. Courville, "Representation mixing for tts synthesis," in International Conference on Acoustics, Speech and Signal Processing (ICASSP), 2019.

[16] A. Graves, "Generating sequences with recurrent neural networks," arXiv preprint arXiv:1308.0850, 2013.

[17] Z. Zeng, J. Wang, N. Cheng, T. Xia, and J. Xiao, "Aligntts: Efficient feed-foward text-to-speech system without explicit alignment," in International conference on acoustics, speech and signal processing (ICASSP), 2020

[18] A. Vaswani, N. Shazeer, N. Parmar, J. Uszkoreit, L. Jones, A. N. Gomez, Ł. Kaiser, and I. Polosukhin, "Attention is all you need," in Advances in neural information processing systems (NIPS), 2017.
[19] Y. Lee and T. Kim, "Robust and fine-grained prosody control of end-to-end speech synthesis," in International conference on acoustics, speech and signal processing (ICASSP), 2019.

[20] G. Sun, Y. Zhang, R. J. Weiss, Y. Cao, H. Zen, and Y. Wu, "Fully-hierarchical fine-grained prosody modeling for interpretable speech synthesis," in International conference on acoustics, speech and signal processing (ICASSP), 2020.

[21] G. Sun, Y. Zhang, R. J. Weiss, Y. Cao, H. Zen, A. Rosenberg, B. Ramabhadran, and Y. Wu, "Generating diverse and natural textto-speech samples using a quantized fine-grained vae and autoregressive prosody prior,' in International Conference on Acoustics, Speech and Signal Processing (ICASSP), 2020.

[22] T. Hayashi, S. Watanabe, T. Toda, K. Takeda, S. Toshniwal, and K. Livescu, "Pre-Trained Text Embeddings for Enhanced Textto-Speech Synthesis," in Annual Conference of the International Speech Communication Association (Interspeech), 2019.

[23] W. Fang, Y.-A. Chung, and J. Glass, "Towards transfer learning for end-to-end speech synthesis from deep pre-trained language models," arXiv preprint arXiv:1906.07307, 2019.

[24] Y. Xiao, L. He, H. Ming, and F. K. Soong, "Improving prosody with linguistic and bert derived features in multi-speaker based mandarin chinese neural tts," in International Conference on Acoustics, Speech and Signal Processing (ICASSP), 2020.

[25] J. Devlin, M.-W. Chang, K. Lee, and K. Toutanova, "Bert: Pretraining of deep bidirectional transformers for language understanding," arXiv preprint arXiv:1810.04805, 2018.

[26] Z. Yang, Z. Dai, Y. Yang, J. Carbonell, R. R. Salakhutdinov, and Q. V. Le, "Xlnet: Generalized autoregressive pretraining for language understanding," in Advances in neural information processing systems (NIPS), 2019.

[27] J. Lee, W. Yoon, S. Kim, D. Kim, S. Kim, C. H. So, and J. Kang, "Biobert: a pre-trained biomedical language representation model for biomedical text mining," Bioinformatics, vol. 36, no. 4, pp. 1234-1240, 2020.

[28] T. Young, D. Hazarika, S. Poria, and E. Cambria, "Recent trends in deep learning based natural language processing," ieee Computational intelligenCe magazine, vol. 13, no. 3, pp. 55-75, 2018.

[29] K. He, X. Zhang, S. Ren, and J. Sun, "Deep residual learning for image recognition," in IEEE conference on computer vision and pattern recognition (CVPR), 2016.

[30] J. L. Ba, J. R. Kiros, and G. E. Hinton, "Layer normalization," arXiv preprint arXiv:1607.06450, 2016.

[31] N. Srivastava, G. Hinton, A. Krizhevsky, I. Sutskever, and R. Salakhutdinov, "Dropout: a simple way to prevent neural networks from overfitting," The journal of machine learning research, vol. 15, no. 1, pp. 1929-1958, 2014.

[32] C. M. Bishop, Mixture density networks. Aston University, 1994.

[33] K. Ito, “The lj speech dataset," https://keithito.com/LJ-SpeechDataset/, 2017.

[34] Databaker (Beijing) Technology Co.,Ltd., "Chinese standard mandarin speech copus," https://www.databaker.com/open_source.html, 2020.

[35] Z. Lan, M. Chen, S. Goodman, K. Gimpel, P. Sharma, and R. Soricut, "Albert: A lite bert for self-supervised learning of language representations," arXiv preprint arXiv:1909.11942, 2019.

[36] D. P. Kingma and J. Ba, "Adam: A method for stochastic optimization," arXiv preprint arXiv:1412.6980, 2014. 